\title{
Role of Hydrophobic Interactions in the Encounter Complex Formation of the Plastocyanin and Cytochrome $f$ Complex Revealed by Paramagnetic NMR Spectroscopy
}

\author{
Sandra Scanu, ${ }^{\dagger}$ Johannes M. Foerster, $^{\ddagger}$ G. Matthias Ullmann, ${ }^{\ddagger}$ and Marcellus Ubbink* ${ }^{\dagger}$ \\ ${ }^{\dagger}$ Institute of Chemistry, Leiden University, Einsteinweg 55, 2333 CC Leiden, The Netherlands \\ ${ }^{\ddagger}$ Structural Biology/Bioinformatics, University of Bayreuth, Universitätsstrasse 30, 95447 Bayreuth, Germany
}

Supporting Information

ABSTRACT: Protein complex formation is thought to be at least a two-step process, in which the active complex is preceded by the formation of an encounter complex. The interactions in the encounter complex are usually dominated by electrostatic forces, whereas the active complex is also stabilized by noncovalent short-range forces. Here, the complex of cytochrome $f$ and plastocyanin, electron-transfer proteins involved in photosynthesis, was studied using paramagnetic relaxation NMR spectroscopy. Spin labels were attached to cytochrome $f$, and the relaxation enhancements of plastocyanin nuclei were measured, demonstrating that a large part of the cytochrome $f$ surface area is sampled by plastocyanin. In contrast, plastocyanin is always oriented

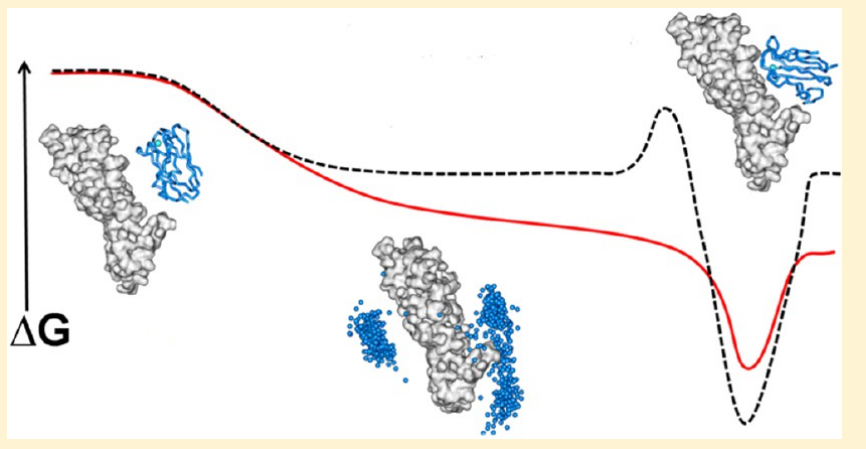
with its hydrophobic patch toward cytochrome $f$. The complex was visualized using ensemble docking, showing that the encounter complex is stabilized by hydrophobic as well as electrostatic interactions. The results suggest a model of electrostatic preorientation before the proteins make contact, followed by the formation of an encounter complex that rapidly leads to electron-transfer active conformations by gradual increase of the overlap of nonpolar surface areas on cytochrome $f$ and plastocyanin. In this model the distinction between the encounter and active complexes vanishes, at least in the case of electrontransfer complexes, which do not require a high degree of specificity.

\section{INTRODUCTION}

A general model for protein-protein interactions describes protein association as a stepwise process in which the formation of the final complex is preceded by that of a transient, lowly populated state encounter complex. ${ }^{1}$ In the first step of association, when the freely diffusing proteins approach each other, they are steered toward certain encounter orientations by long-range electrostatic interactions. In the encounter state, proteins still show few specific interactions. They rather tend to assume multiple orientations to sample the surface of the partner and reduce the dimensionality of the search for the specific binding site. ${ }^{2}$ The final complex is dominated by shortrange, specific interactions, which stabilize it in a single orientation. An encounter complex will not always proceed toward the final complex. In some cases it is futile and will dissociate again. ${ }^{3,4}$ The applicability of this model has been theoretically and experimentally demonstrated for complexes with electrostatic-assisted association, both when the interaction partners showed high overall charge complementarity 5,6 and when opposite charges are more localized in specific regions on the surface of the reactants. ${ }^{7,8}$ At the same time, given the wide variety in the electrostatic surface properties of proteins, this model cannot readily be generalized for all protein complexes. For complexes in which the interaction partners do not present charge complementarity or apparent dipolar interactions, a desolvation-mediated association has been suggested on the basis of theoretical work, ${ }^{9,10}$ in which hydrophobic interactions guide both encounter complex formation and stabilization of the final complex. Experimental evidence of a predominantly hydrophobic-driven binding event is rare, although some has been reported. ${ }^{11,12}$

To investigate the contribution of the different forces involved in the molecular recognition process for transient complexes involved in electron transfer (ET), the complex formed by plastocyanin (Pc) and cytochrome $f(\mathrm{Cyt} f$ ) from the cyanobacterium Nostoc sp. PCC 7119 was studied. Pc and Cyt $f$ are redox partners in oxygenic photosynthesis in plants, green algae, and cyanobacteria. Pc transfers electrons from Cyt $f$ of the cytochrome $b_{6} f$ complex to photosystem I (PSI). ${ }^{13}$ In both proteins the redox active sites are buried below extensive hydrophobic surface patches, which form the specific binding site. The overall electrostatic properties of the proteins vary significantly between different species and influence the final

Received: February 12, 2013

Published: April 29, 2013 
orientation of the complex. In plants, the final complex has been shown to be electrostatically stabilized in a "side-on" orientation by complementary localized charges on the protein surfaces, negative in $\mathrm{Pc}$ and positive in Cyt $f^{14,15}$ Complementary charges tilt Pc toward the long side of Cyt $f$ and align the hydrophobic binding sites, thus facilitating the ET reaction. In the cyanobacterium Phormidium laminosum, the final complex assumes a "head-on" orientation. ${ }^{12} \mathrm{Pc}$ is oriented perpendicular to the heme plane and comes into contact with Cyt $f$ only with the hydrophobic patch. The "side-on" orientation was also observed in the complexes from the cyanobacteria Nostoc ${ }^{16}$ and Prochlorothrix hollandica, ${ }^{17}$ in which the charges are inverse compared to the plant counterparts, being positive in $\mathrm{Pc}$ and negative in Cyt $f$. Site-directed mutagenesis of key interface residues important for the overall electrostatic potential of the proteins from Nostoc demonstrated that electrostatic interactions heavily regulate the kinetics of complex formation. ${ }^{18,19}$ Interestingly, the loss of negatively charged residues in Cyt $f$, in which charges are spread over a large part of the surface instead of being localized in a specific region, caused only small changes of the association rate constant, ${ }^{19}$ whereas mutations of positively charged residues in a conserved region in Pc showed these charges to be fundamental for fast association. ${ }^{18}$ The surface charge properties of Pc and Cyt $f$ appear to influence the degree of dynamics within the complexes. ${ }^{20}$ The Ph. laminosum and Pr. hollandica complexes showed to be highly dynamic. ${ }^{12,17}$ The solution structures of the above-mentioned complexes have been obtained by taking advantage of the pseudocontact shift (PCS) caused by the paramagnetic heme iron of Cyt $f$ on backbone amide protons of Pc. PCS from the heme are not very sensitive to dynamics, although the presence of many different Pc orientations will cause a decrease in the observed average PCS. In a recent paramagnetic relaxation enhancement (PRE) NMR study on the Nostoc system, we demonstrated that the Pc-Cyt $f$ complex is more dynamic than was suggested by PCS, indicating the presence of a significantly populated encounter state. $^{21}$

PRE NMR spectroscopy has proven to be a sensitive technique for the detection and visualization of lowly populated intermediates in protein-DNA ${ }^{22}$ and protein-protein complexes. $^{8,23,24}$ PREs arise from magnetic dipolar interactions between the unpaired electron of a paramagnetic center and the observed nucleus, which causes an increase in the relaxation of the latter. Due to the large magnetic moment of the unpaired electron and the inverse sixth power distance dependence of the PRE, it is very large for nuclei that spend time in close proximity of the paramagnetic center. The sensitivity of PRE for lowly populated states is due to the fact that in the NMR fast exchange regime the observed PRE rate is a population weighted average of all species present in solution. ${ }^{25}$ If in the lowly populated state the nucleus is close to the paramagnetic center, a PRE can be detected on the exchange averaged signal, even if the population is as low as $1 \%$. Intermolecular PREs can thus be used to investigate transient intermediates in proteinprotein complexes. ${ }^{23}$ The observed PREs provide explicit qualitative evidence of the presence of the encounter state, but they do not provide a complete description of the encounter complex. Furthermore, the visualization of the encounter complex is an 'inverse' problem since many possible solutions can correspond to the observed PREs. To depict the encounter complex, experimental data need to be supported by theoretical models, generated by computational approaches. In the ensemble docking approach, multiple conformers of a protein are simultaneously docked to the other protein on the basis of the experimental PRE. ${ }^{23}$ The encounter complex is visualized as an ensemble of orientations that fit the experimental restraints. In purely theoretical methods, such as Brownian dynamics (BD) and Monte Carlo (MC) simulations, in which proteins are docked only on the basis of electrostatic interactions, ${ }^{7,26}$ the encounter complex is given as a distribution of the favorable electrostatic orientations. ${ }^{8}$

In our previous study on the Nostoc complex, ${ }^{21}$ three spin labels were attached to Cyt $f$ at sites surrounding the binding site for plastocyanin. It was demonstrated that those spin labels did not affect the Pc chemical shift perturbation (CSP) map caused by binding to Cyt $f$. The PRE data were not in agreement with a single binding orientation of $\mathrm{Pc}$, because the affected amide groups on Pc were very similar for each of the spin labels, despite their different locations on Cyt $f$. This finding showed that $\mathrm{Pc}$ was sampling multiple orientations within the complex. To map the range of the encounter orientations of $\mathrm{Pc}$, in the present study the number of spin label positions was extended to nine. The results were used to visualize the encounter complex by ensemble docking, and this model was compared with MC simulations. The comparison indicates that long-range electrostatic interactions preorient $\mathrm{Pc}$ with the hydrophobic patch toward Cyt $f$ and that Pc maintains the same orientation while sampling the surface of Cyt $f$. The ensemble docking visualization of the encounter complex showed that the encounter complex is stabilized by hydrophobic as well as electrostatic interactions. We propose a model for Pc-Cyt $f$ complex formation in which long-range electrostatic interactions preorient the unbound proteins before they make contact. Pc diffusively binds to Cyt $f$ forming an extended encounter complex stabilized by the overlap of the respective nonpolar surface areas, and the encounter complex rapidly evolves to ET active conformations. The ambiguous distinction between the encounter and the active complex in this system will be discussed in the context of the physiological cytochrome $b_{6} f$ complex.

\section{EXPERIMENTAL SECTION}

Protein Production and Purification. ${ }^{15} \mathrm{~N}$ enriched, $\mathrm{Zn}$ substituted Pc was produced and purified as described before. ${ }^{21}$ The concentration of the protein was determined by absorbance spectroscopy using $\varepsilon_{280}=5 \mathrm{mM}^{-1} \mathrm{~cm}^{-1}$. The yield of pure protein was $10 \mathrm{mg} /$ $\mathrm{L}$ of culture.

The pEAF-WT plasmid, containing the gene of the soluble domain (residue 1-254) of Nostoc sp. PCC7119 Cyf $f$ was kindly provided by Prof. Dr. Miguel A. De la Rosa (University of Seville). Cyt $f$ mutants were obtained using pEAF-WT plasmid as template for mutagenesis. The mutations to cysteine were introduced by using the QuikChange Site-Directed Mutagenesis kit (Stratagene). The primers used for the mutations at the positions N71, Q104, and S192 were described before. $^{21,27}$ The primers employed for the introduction of a cysteine at the positions Q7, Q38, A63, Q125, S181, and Q242 are reported in Table S1.

Truncated Cyt $f$ was produced in E. coli MV1190 (D(lac-proAB), thi, sup $\mathrm{E}, \mathrm{D}(\operatorname{srl}-\operatorname{rec} \mathrm{A}) \quad 306:$ Tn $10\left(\operatorname{tet}^{\mathrm{r}}\right)\left[\mathrm{F}^{\prime}: \operatorname{tra} \mathrm{D} 36\right.$, pro $\mathrm{AB}+$, lac $\left.\left.\mathrm{I}^{\mathrm{q}} \mathrm{Z} \Delta \mathrm{M} 15\right]\right)$, transformed with pEAF-WT or mutant plasmids, and cotransformed with $\mathrm{pEC} 86$, containing a cassette for $c$-type cytochrome overexpression. ${ }^{28}$ Production and purification of the protein and spin label attachment of (1-acetoxy-2,2,5,5-tetramethyl- $\delta$ 3-pyrroline-3-methyl) methanethiosulfonate (MTS) or (1-oxyl-2,2,5,5tetramethyl- $\delta$-3-pyrroline-3-methyl) methanethiosulfonate (MTSL) were performed as previously reported. ${ }^{18,21}$ The yield of protein production ranged from 1 to $2 \mathrm{mg} / \mathrm{L}$ of culture. The expression of 
Q125C mutant was not reproducible, and only a small amount of protein was obtained and used for NMR experiments. The concentration of the protein was determined by absorbance spectroscopy using $\varepsilon_{556}=31.5 \mathrm{mM}^{-1} \mathrm{~cm}^{-1}$ for ferrous Cyt $f$.

NMR Experiments. All NMR samples contained MES $(20 \mathrm{mM}$, $\mathrm{pH} 6)$ and $6 \% \mathrm{D}_{2} \mathrm{O}$ for lock. The ferric state of Cyt $f$ was preserved by addition of $\mathrm{K}_{3}\left[\mathrm{Fe}(\mathrm{CN})_{6}\right](50 \mu \mathrm{M})$. The $\mathrm{pH}$ of the sample was adjusted with small aliquots of $\mathrm{HCl}(0.5 \mathrm{M})$ and $\mathrm{NaOH}(0.5 \mathrm{M})$. For the chemical shift perturbation experiments Cyt $f$ was titrated into $\mathrm{Zn}$ substituted ${ }^{15} \mathrm{~N}$ Pc $(50 \mu \mathrm{M})$. Spectra were recorded at multiple Cyt $f:$ Pc molar ratios $(0.1,0.2,0.4,0.6,0.8,1.0,2.5,5.0)$. CSP experiments were not performed for Q125C Cyt $f$ because of lack of protein. Samples for PRE measurements contained $33 \mu \mathrm{M}$ Cyt $f$ for the Q125C mutant and $66 \mu \mathrm{M}$ for the other mutants, labeled with either MTS or MTSL. Samples also contained Zn-substituted ${ }^{15} \mathrm{~N}$ Pc, $100 \mu \mathrm{M}$ in the complex with Q125C Cyt $f$ and $200 \mu \mathrm{M}$ for the other Cyt $f$ mutants. All NMR spectra were recorded at $298 \mathrm{~K}$ on a Bruker Avance III 600 $\mathrm{MHz}$ spectrometer equipped with a TCI-Z-GRAD Cryoprobe. The ${ }^{1} \mathrm{H}-{ }^{15} \mathrm{~N}$ HSQC spectra were acquired with 1024 and 80 complex points in the direct and indirect dimensions, respectively.

NMR Data Analysis. The NMR spectra were processed with NmrPipe $^{29}$ and analyzed with CcpNMR Analysis. ${ }^{30}$ Chemical shift perturbation analysis was carried out as described before. ${ }^{21}$

The PREs were determined according to the procedure described by Battiste and Wagner. ${ }^{31}$ The intensity ratio $I_{p} / I_{d}$ of the Pc resonances in the presence of MTSL-Cyt $f\left(I_{p}\right)$ and MTS-Cyt $f\left(I_{d}\right)$ was normalized by dividing them by the average value of the 10 largest $I_{p} / I_{d}$ values $(1.28$ for $\mathrm{Q} 7 \mathrm{C}, 1.63$ for $\mathrm{Q} 38 \mathrm{C}, 1.16$ for $\mathrm{A} 63 \mathrm{C}, 1.13$ for N71C and Q104C, 1.37 for Q125C, 0.83 for S181C, and 1.06 for S192C and 0.92 for Q242C). The PRE $\left(\Gamma_{2}\right)$ values were calculated according to the formula:

$$
\frac{I_{p}}{I_{d}}=\frac{R_{2 \mathrm{~d}} \exp \left(-\Gamma_{2} t\right)}{R_{2 \mathrm{~d}}+\Gamma_{2}}
$$

$R_{2 \mathrm{~d}}$ represents the transverse relaxation rate in the diamagnetic sample, which was calculated from the line width at half height obtained from a Lorentzian peak fit in the direct dimension, by using FUDA (this software was kindly provided by Dr. D. Fleming Hansen, University College London). The symbol $t$ indicates the time for transverse relaxation during the pulse sequence $(9 \mathrm{~ms})$. The $\Gamma_{2}$ values were extrapolated to the $100 \%$ bound state using the experimentally obtained $K_{\mathrm{D}}$

Monte Carlo Simulations of the Encounter Complex. The structure of the soluble part of Cyt $f$ (residues 1-254) used for the calculation was taken from the crystal structure of the cytochrome $b_{6} f$ complex from Nostoc sp. PCC 7120, PDB entry 2ZT9. ${ }^{32}$ The amino acidic sequences of Cyt $f$ from Nostoc sp. PCC 7120 and sp. PCC 7119 are identical. The structure file for Pc was taken from the PDB entry 2GIM. ${ }^{33}$ The hydrogen atoms were added with the module HBUILD $^{34}$ of Charmm. ${ }^{35}$ To preserve the original structure, only the hydrogen atoms were minimized with the Charmm force field, ${ }^{36}$ while the other atoms were kept fixed in their original position. The iron of Cyt $f$ and the copper of Pc were considered to be in the oxidized state, like in the experiments. The electrostatic potentials for the single proteins were calculated with $\mathrm{APBS}^{37}$ The dielectric constants for Cyt $f$ and the water were set to 4 and 80 , respectively. For all electrostatic potentials, a box with a diameter of $225 \AA$ in $x, y$, and $z$ directions, with Cyt $f$ centered at the origin of the coordinates frame, was defined. The ionic strength was set to $0.02 \mathrm{M}$ and the temperature to $298 \mathrm{~K}$. The electrostatic potential was calculated with the linearized Poisson-Boltzmann equation.

The docking was performed with the program MC-Dock ${ }^{26}$ and was carried out in a similar way as was done before. ${ }^{8}$ Cyt $f$ was chosen as the receptor, and Pc was the ligand to dock. The simulation consisted of 250 runs with $1 \times 10^{6}$ steps each and was carried out at a temperature of $298 \mathrm{~K}$. Only structures that respected the Metropolis $\mathrm{MC}$ criterion $^{38}$ were saved resulting in about $2.3 \times 10^{6}$ Cyt $f$-Pc orientations. The main difference to the previous simulation consisted in the use of an inclusion grid. The inclusion grid was created by defining a grid with a distance to the surface of Cyt $f$ of $3 \AA$ and a grid point separation of $0.5 \AA$. If any atom of Pc is located within this inclusion grid, the structure was included in the final encounter ensemble, otherwise the orientation was not considered. An ensemble of $5000 \mathrm{Pc}$ orientations, randomly selected, was considered for the calculations. The averaged distances were derived from the ensemble and compared to the experimental distances.

Ensemble Docking. Mutations and spin labels were modeled on the structure of Cyt $f$ (PDB entry 2ZT9), ${ }^{32}$ and four conformations were used to represent the mobility of the spin label. ${ }^{39}$ The structure of Pc was taken from PDB entry 2GIM. ${ }^{33}$

The $\Gamma_{2}$ were converted into distances for structure calculations using eq 2 :

$$
r=\sqrt[6]{\frac{\gamma^{2} g^{2} \beta^{2}}{20 \Gamma_{2}}\left(4 \tau_{c}+\frac{3 \tau_{c}}{1+\omega_{h}^{2} \tau_{c}^{2}}\right)}
$$

Where $r$ is the distance between the oxygen atom of MTSL and the Pc amide proton, $\gamma$ is the proton gyromagnetic ratio, $g$ is the electronic $g$ factor, $\beta$ is the Bohr magneton, $\omega_{h}$ is the Larmor frequency of the proton, and $\tau_{\mathrm{c}}$ is the rotational correlation time of the MTSL oxygenproton vector. $\tau_{\mathrm{c}}$ was taken to be $30 \mathrm{~ns}$ on the basis of the HYDRONMR $^{40}$ prediction of the rotational correlation time for the Pc-Cyt $f$ complex.

The restraints for the calculations were obtained according to eq 3 :

$$
\begin{gathered}
\Gamma_{2}^{\mathrm{obs}}=f_{1} \Gamma_{2}^{\mathrm{ens}}+f_{2} \Gamma_{2}^{\mathrm{final}} \\
f_{1}+f_{2}=1
\end{gathered}
$$

The ensemble $\Gamma_{2}\left(\Gamma_{2}{ }^{\text {ens }}\right)$ was calculated as the difference between observed $\Gamma_{2}\left(\Gamma_{2}{ }^{\text {obs }}\right)$ and back-calculated $\Gamma_{2}$ from the model of the final complex (model 1, PDB entry 1TU2) $\left(\Gamma_{2}^{\text {final }}\right)$. The calculations were carried out with $f_{2}$ values of $=0,0.15,0.25,0.35,0.5,0.65,0.75,0.85$, 0.95 , and 1 . The restraints were grouped into three classes as described before. $^{21}$

A description of the encounter complex was obtained with restrained rigid-body docking in Xplor-NIH 2.9.9 ${ }^{41}$ to minimize the difference between observed and back-calculated distances for all spin labels. Calculations were carried out using either a single Pc conformer or an ensemble of Pc molecules, with between 2 and 20 copies. The distances $(r)$ between an amide proton and the oxygen atom of MTSL were $r^{-6}$ averaged for all MTSL orientations and all Pc conformers. Cyt $f$ and Pc were both considered as rigid bodies, the coordinates of Cyt $f$ were fixed, and Pc ensemble members were allowed to move individually in a restrained rigid-body molecular dynamics calculation. Overlap of Pc copies was allowed, since the ensemble represents a distribution of states. Similarly, overlap of MTSL conformers with other MTSL or Pc copies was allowed. For the visualization of the final encounter complex ensemble 150 dockings were performed, yielding 144 ensembles of $7 \mathrm{Pc}$ conformers, with a difference in the total restraint energy $\leq 20 \%$.

The ensembles from separate dockings were evaluated by calculating the average violation over all experimental distances. Class 1 and 3 restraints are not easily expressed in a $Q$ value. Violations provide a better representation of the fit of all three classes of restraints. Class 2 violations were defined as the absolute difference between experimental and calculated distances for a certain amide nucleus. Class 1 and 3 violations were defined as that difference only for back-predicted distances that were above $14 \AA$ and below $23 \AA$, respectively. The ensemble violation is the average violation for all residues and all spin labels.

\section{RESULTS}

Introduction of Paramagnetic Probes on Cyt $f$. To determine the extent of surface area of Cyt $f$ being sampled by Pc in the encounter complex, 9 cysteine mutants of Cyt $f$ were made for the attachment of nitroxy spin labels. The Cyt $f$ mutants were created for the positions Q7, Q38, A63, N71, 

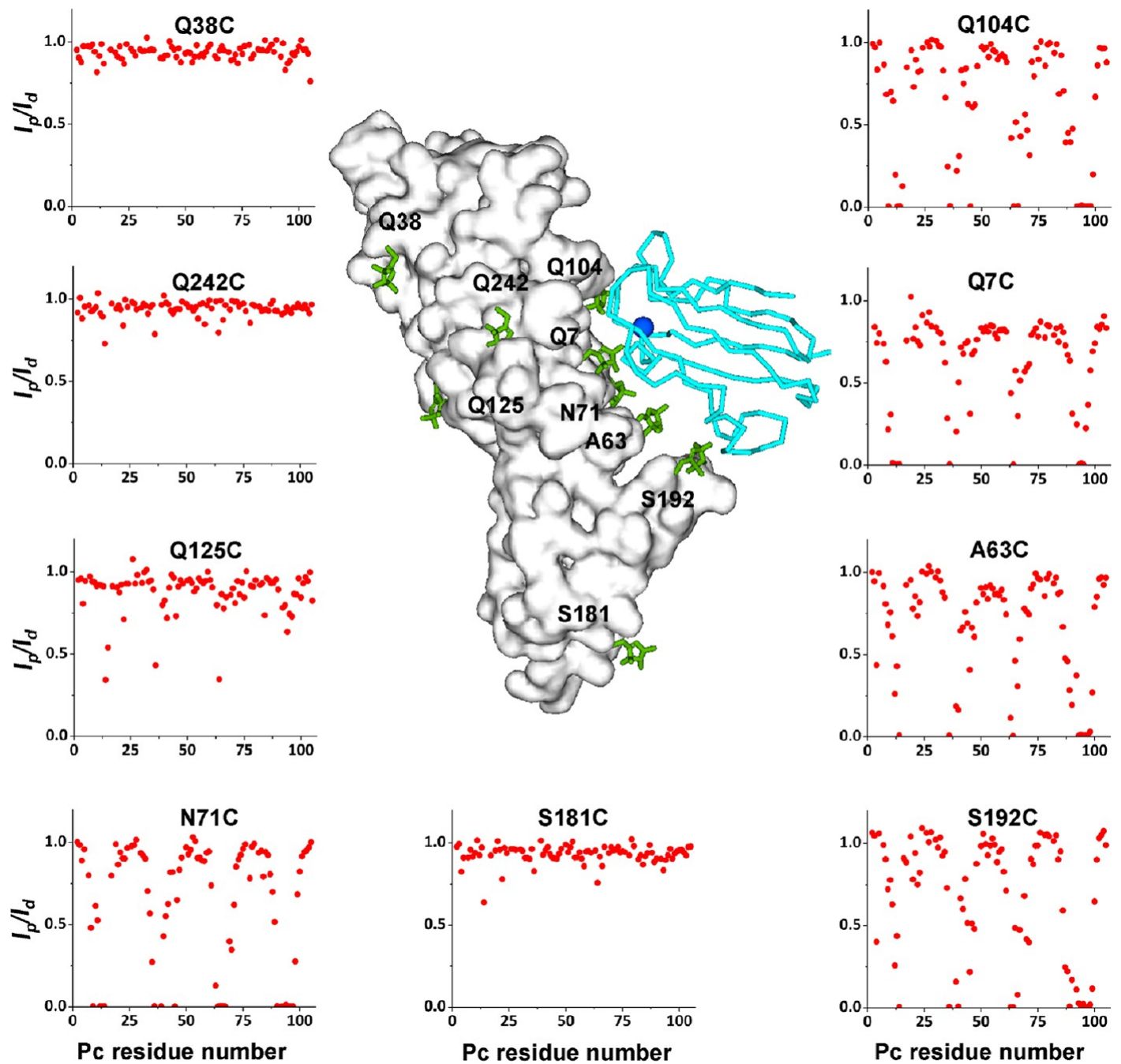

Figure 1. PRE in the Pc-Cyt $f$ complex. Central panel. Location of the spin labels (green sticks) modeled on the Nostoc sp. PCC 7119 Pc-Cyt $f$ complex (PDB entry 1TU2, model 1). ${ }^{16} \mathrm{Pc}$ is shown in cyan $\mathrm{C}_{\alpha}$ trace and the copper as a blue sphere. Cyt $f$ is shown as white surface. This image and others of molecular structures were made with Discovery Studio Visualizer 2.5 (Accelrys). Side panels. The $I_{p} / I_{d}$ ratios (red dots) are plotted against the $\mathrm{Pc}$ residue number for each of the spin label position on Cyt $f$.

Q104, Q125, S181, S192, and Q242. The mutation sites Q7, A63, N71, Q104, and S192 are near to the Pc binding site indicated by the solution model, ${ }^{16}$ whereas the remaining four mutations are located elsewhere (Figure 1, central panel). To preserve the original electrostatic potential of Cyt $f$, only polar, uncharged amino acids and one Ala were selected for mutation to cysteine. The copper in ${ }^{15} \mathrm{~N}$ Pc was substituted by $\mathrm{Zn}^{\mathrm{II}}$ to eliminate the paramagnetic effect and possible interference of the ET reaction caused by the presence of $\mathrm{Cu}^{\mathrm{II}}{ }^{42}$

To test whether the presence of spin label interferes with the Pc-Cyt $f$ binding, CSP analysis was performed for Pc bound to Cyt $f$ wild-type and mutants conjugated to the diamagnetic control label MTS. Cyt $f$ was thus titrated into a solution of ${ }^{15} \mathrm{~N}$ $\mathrm{Zn}-\mathrm{Pc}$ and HSQC spectra were acquired at each titration point. The CSP curves for the most affected residues were fitted to obtain a dissociation constant for each complex (Figure S1). The $K_{\mathrm{D}}$ values are listed in Table 1 .

The $K_{D}$ value for the wt complex of $8 \pm(3) \times 10^{-5} \mathrm{M}$ is similar to the reported values of $4 \times 10^{-5} \mathrm{M}$ for $\mathrm{Cu}-\mathrm{Pc}^{43}$ and 6 $\times 10^{-5} \mathrm{M}$ for Cd-substituted Pc. ${ }^{43}$

Most of Cyt $f$ variants yielded $K_{\mathrm{D}}$ values within the experimental error of that of the wild-type. Moreover, the
Table 1. Dissociation Constants of the Complexes Formed by Nostoc Zn-Pc with Wild-Type and MTS-Conjugated Cyt $f^{a}$

$\begin{array}{cc}\text { Cyt } f \text { mutant } & K_{\mathrm{D}}\left(10^{-5} \mathrm{M}\right) \\ \text { wild-type } & 8(3) \\ \text { Q7C-MTS } & 5(1) \\ \text { Q38C-MTS } & 2(1) \\ \text { A63C-MTS } & 2(1) \\ \text { N71C-MTS } & 4(1) \\ \text { Q104C-MTS } & 3(1) \\ \text { S181C-MTS } & 6(2) \\ \text { S192C-MTS } & 4(1) \\ \text { Q242C-MTS } & 9(2)\end{array}$

${ }^{a}$ The errors are indicated in parentheses and represent the precision of the fit.

binding maps, obtained by coloring the protein residues according to the size of CSP, present a pattern similar to the wild-type, indicating that the mutations and the attachment of MTS at these positions cause no significant effects on the affinity of $\mathrm{Pc}$ for Cyt $f$ and orientation of $\mathrm{Pc}$ with the respect to Cyt $f$ in the complex. In each case Pc binds predominantly via 
A

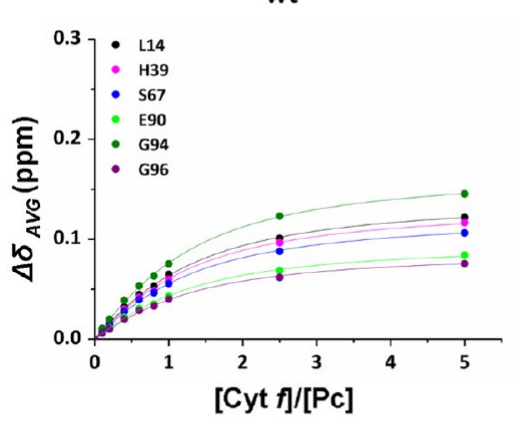

B

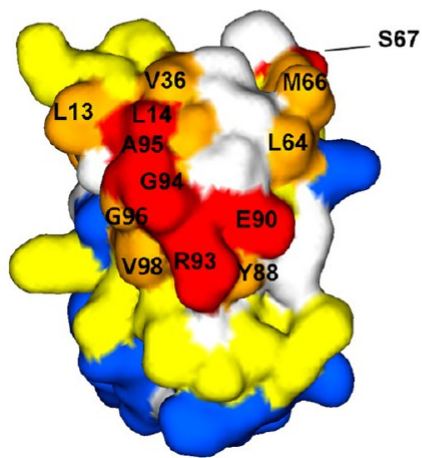

Q38C
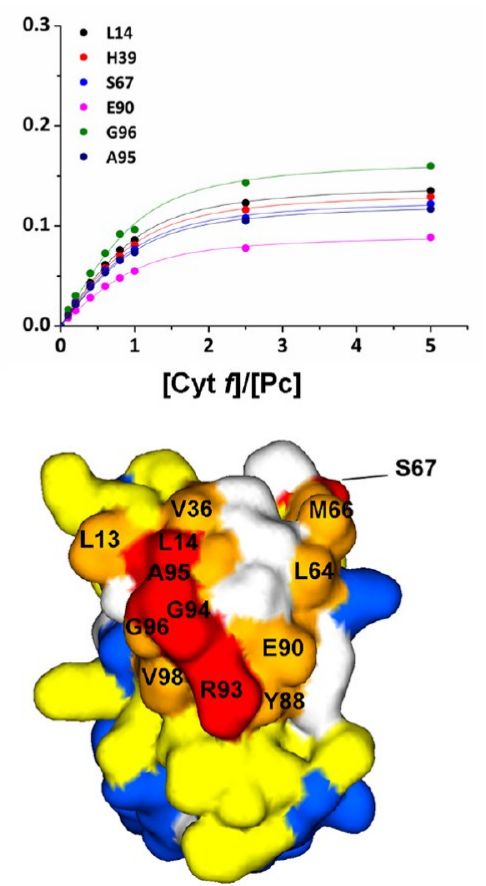

A63C
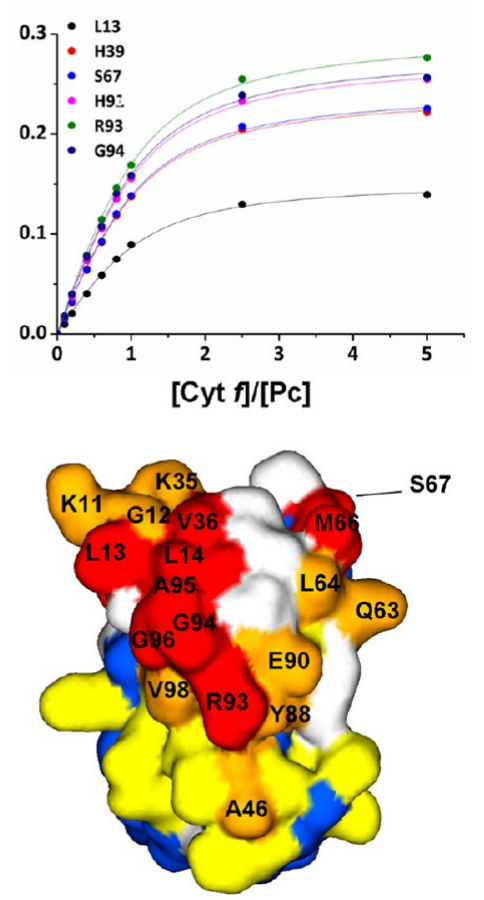

Figure 2. The interaction of Nostoc Zn-substituted Pc with wild-type Cyt $f$ and Q38C and A63C MTS-conjugated variants. (A) Binding curves for selected residues were fitted globally to a 1:1 binding model. ${ }^{21}$ (B) Chemical shift perturbation maps color-coded on a surface model of Pc (PDB entry $2 \mathrm{GIM}){ }^{33}$ with red, $\Delta \delta_{\text {avg }} \geq 0.10 \mathrm{ppm}$; orange, $\Delta \delta_{\text {avg }} \geq 0.05 \mathrm{ppm}$; yellow, $\Delta \delta_{\text {avg }} \geq 0.02 \mathrm{ppm}$; and blue, $\Delta \delta_{\text {avg }}<0.02 \mathrm{ppm}$. Prolines and residues with overlapping resonances are in white.

the hydrophobic patch and the region around Arg 93 (Figure S2), similarly to previously reported data on Cd-substituted Pc in the presence of reduced Cyt $f .{ }^{16}$ The Q38C-MTS and A63CMTS Cyt $f$ variants exhibited $K_{\mathrm{D}}$ values of $2 \pm(1) \times 10^{-5} \mathrm{M}$, which represent a small increase of binding affinity. In the case of Q38C-MTS Cyt $f$, the average size of the CSP and binding map were similar to wild-type. The spin label position is located far from the final binding site (Figure 1), so the reason for the lower $K_{\mathrm{D}}$ values remains unclear. Larger perturbations of the resonance positions were observed for binding of Pc to Cyt $f$ A63C-MTS than in the other studied cases. The largest CSPs were about twice as large as those in the presence of wild-type Cyt $f$ (Figure 2A). Interestingly, the binding map is still similar to that of wt, although the effects of binding are stronger (Figure 2B). Under the assumption that CSPs predominantly represent the final state, ${ }^{44,45}$ this observation suggests that Pc binds Cyt $f$ A63C-MTS in the same orientation as wt Cyt $f$ but that the final state is more populated and the encounter state less.

Paramagnetic Relaxation Enhancements. To determine whether PREs could arise from unspecific interactions, free MTS (diamagnetic) and MTSL (paramagnetic), with a concentration corresponding to that of spin labeled Cyt $f(66$ $\mu \mathrm{M}$, see below), were mixed with ${ }^{15} \mathrm{~N}$-labeled $\mathrm{Zn}$-Pc $(200 \mu \mathrm{M})$. No line broadening of the resonances was observed in the presence of the paramagnetic spin label, indicating that unspecific interactions with the label are not significant under these conditions.

Then, MTSL was attached to each of the nine Cyt $f$ mutants, and the tagged proteins were titrated to Pc to a molar ratio of 1:0.3 for Pc:Cyt $f$. At this ratio, the average fraction of Pc bound to Cyt $f$ is $24 \%$. The CSP studies indicated that association and dissociation are in the fast exchange regime, so the observed
PREs are a weighted average of free Pc, encounter complex and final complex. Thus, the PREs can be extrapolated to the $100 \%$ bound state (encounter complex + final complex) by dividing by the fraction of bound Pc.

Spin labels attached to Cyt $f$ near the binding site for Pc in the final complex, namely at the positions Q7, A63, N71, Q104, and S192, caused an extensive broadening of Pc resonances, reflected in a decrease of the $I_{p} / I_{d}$ ratio, the ratio of peak intensities in the spectra of the paramagnetic and diamagnetic samples (Figure 1). Surprisingly, mutants with the spin label located on the backside of Cyt $f$ relative to the binding site also yielded moderate to strong PREs. For three of these mutants, Q38C, S181C, and Q242C, only moderate effects were observed, whereas the spin label at position 125 caused strong PRE on two Pc residues, L14 and L64, which are part of the hydrophobic patch. From the $I_{p} / I_{d}$ ratios, the PRE $\left(\Gamma_{2}\right)$ were determined and extrapolated to $100 \%$ bound Pc. The PREs were mapped on the surface of Pc, shown in Figure 3.

The PRE patterns observed in the presence of spin label attached near the main binding site are very similar. This result is surprising, because the labels are located on different sides of Cyt $f$ in the structure of the final complex as shown in Figure 1, and thus it is expected that different regions of Pc would be affected. The results suggest that Pc samples an extensive area of Cyt $f$ predominantly with one face oriented toward it, since no strong PREs were observed on the back-side of Pc (Figure S3). The maps also resemble the CSP maps in Figure 2, confirming that the hydrophobic patch and the region around Arg 93 are the interaction sites of Pc in the complex with Cyt $f$. The comparison of the PRE maps with the charge distribution map of Pc (Figure 3, top left) indicates that among the residues, which experience most PRE, only the minority is negatively (E90) or positively (K11, K35, and R93) charged, 

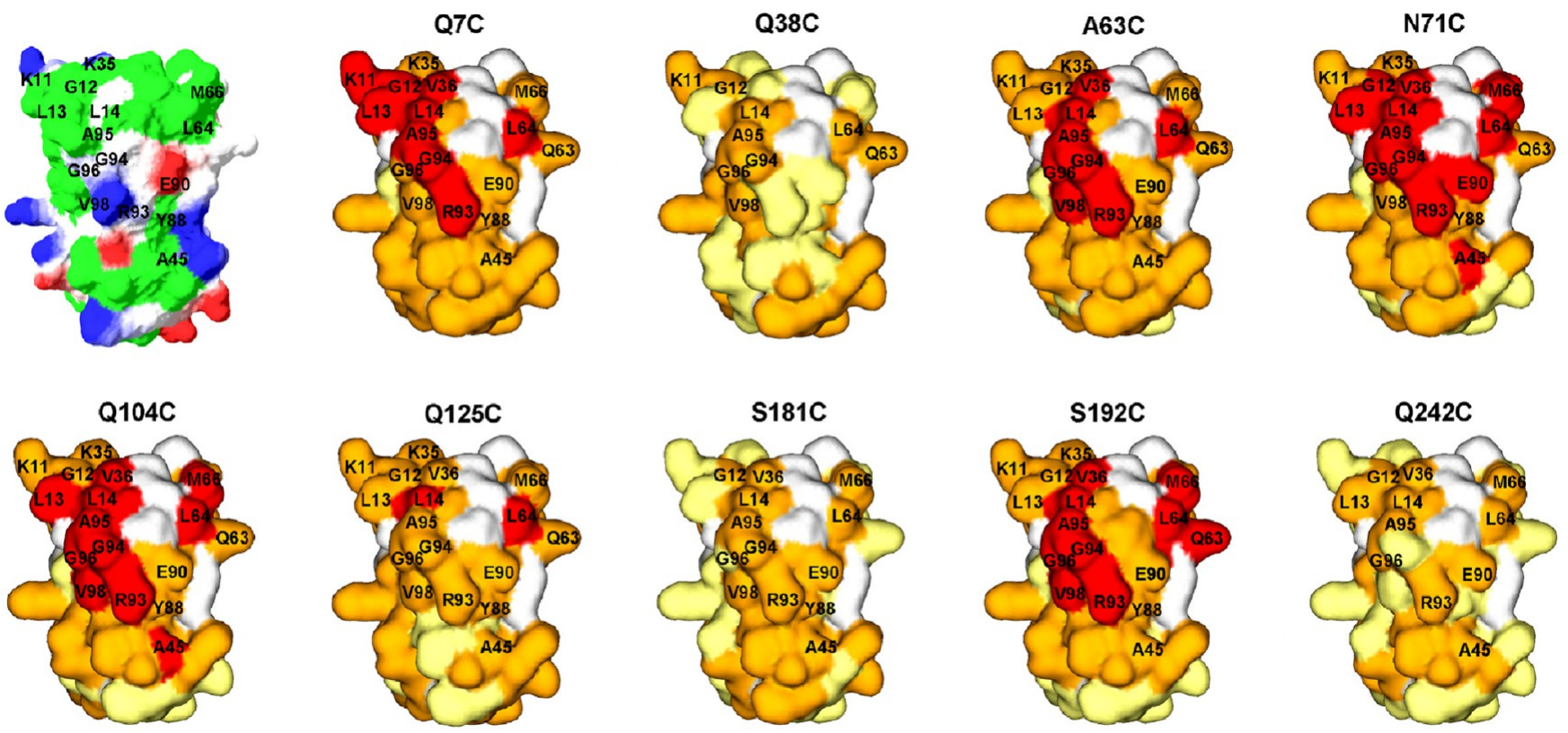

Figure 3. PRE maps of Zn-substituted Pc bound to MTSL-conjugated Cyt $f$, color-coded on a surface model of Pc (PDB entry 2GIM). The sites of spin label attachment are indicated in Figure 1, central panel. Red, $\Gamma_{2} \geq 200 \mathrm{~s}^{-1}$; orange, $10 \mathrm{~s}^{-1}<\Gamma_{2}<200 \mathrm{~s}^{-1}$, and yellow $\Gamma_{2} \leq 10 \mathrm{~s}^{-1}$. Prolines and residues with overlapping resonances are white. Top left, the charge distribution of Pc with negatively and positively charged side chains shown in red and blue, respectively. Hydrophobic side chains are shown in green, and polar side chains are in white.

whereas the majority has a hydrophobic nature. Residues L13, L14, V36, L64, A95, and V98 are part of the hydrophobic patch, which also represents the main binding site and the likely site for ET. ${ }^{16}$

MC Simulations. Visualization of the encounter state on the basis of the PRE data is not straightforward, because the data represent a weighted average of all orientations of Pc within the complex, and thus, an infinite number of ensembles can produce the experimental data set. The encounter complex of Cyt $c$ and Cyt $c$ peroxidase was successfully visualized by combining PRE data and rigid-body MC simulations, ${ }^{8}$ showing that the formation of this encounter complex is solely driven by electrostatic interactions. In MC docking, a mobile protein is docked to a target molecule under the influence of an electrostatic field and MC sampling. ${ }^{26}$ In this way, chargecharge interactions represent the only force that brings together the proteins. Following the same rationale, MC simulations for Pc-Cyt $f$ complex were performed, and the Boltzmann distribution of orientations of Pc in complex with Cyt $f$, and vice versa, was obtained. The centers-of-mass of Pc (Figure S4A) and Cyt $f$ (Figure S4B) are shown as blue and green spheres, respectively, around the interaction partner, shown as surface model. In the MC ensemble Pc is widely spread over the surface of Cyt $f$ in correspondence with the negative charges distribution. Cyt $f$ is overall negative with most charges in the region surrounding the heme on the large domain and lower charge density on the surface opposite of the heme. These results are inconsistent with the PREs observed in the presence of spin labels located at positions far from the heme, such as Q125 (backside) and S181C (small domain). The distribution of Cyt $f$ around Pc is off-center from the CSP map obtained for binding to Cyt $f$. Thus, qualitatively the MC ensembles are not in complete agreement with the experimental data.

The observed PREs result from the contributions of both the encounter and the final complexes, whereas the MC ensemble is assumed to represent mostly the encounter state. To separate the PRE contributions of the two states, the PREs from the solution model of the final complex were back-calculated and subtracted from experimental PREs assuming a population of the final state $\left(f_{2}\right)$ varying from 0 to 1 . The resulting PREs represent the encounter state at decreasing population, and these were converted into distances and compared with the average distances calculated from the MC ensemble (see Experimental Section for details). Ensembles composed of the $100,1000,2500$, and 5000 randomly selected structures were considered for the analysis. Independent of the size of the MC ensemble and of the population of the two states, no good match with experimental data was found (Figure S5). These findings suggest that the formation of the $\mathrm{Pc}-\mathrm{Cyt} f$ encounter complex is not exclusively driven by electrostatic forces. Other contributions must play a significant role, and therefore, $\mathrm{MC}$ simulations cannot provide a complete description of this encounter complex.

Ensemble Docking. The quantitative interpretation of PRE for the visualization of transient encounter complexes requires the use of PRE restraints in docking calculations. ${ }^{25} \mathrm{An}$ ensemble of orientations that represents the encounter state and agrees with the data is generated by docking several conformers of a protein ( $\mathrm{Pc}$ in this work) simultaneously while minimizing the difference between the back-calculated PRE averaged over all conformers and the experimental data. This procedure is repeated many times, and because many different ensembles fit the experimental data, the result is a 'cloud' of orientations. $^{23,46-51}$ An ensemble of non-interacting Pc structures was generated, and docking calculations were performed with PRE restraints arising from all nine spin labels at the same time. Though variants Cyt $f$ Q38- and A63C-MTSL appeared to have some influence on the affinity or the equilibrium between encounter state and final complex (see above), they were included in the calculations, because we found that the description of the encounter complex was similar, whether or not these restraints were included in the 

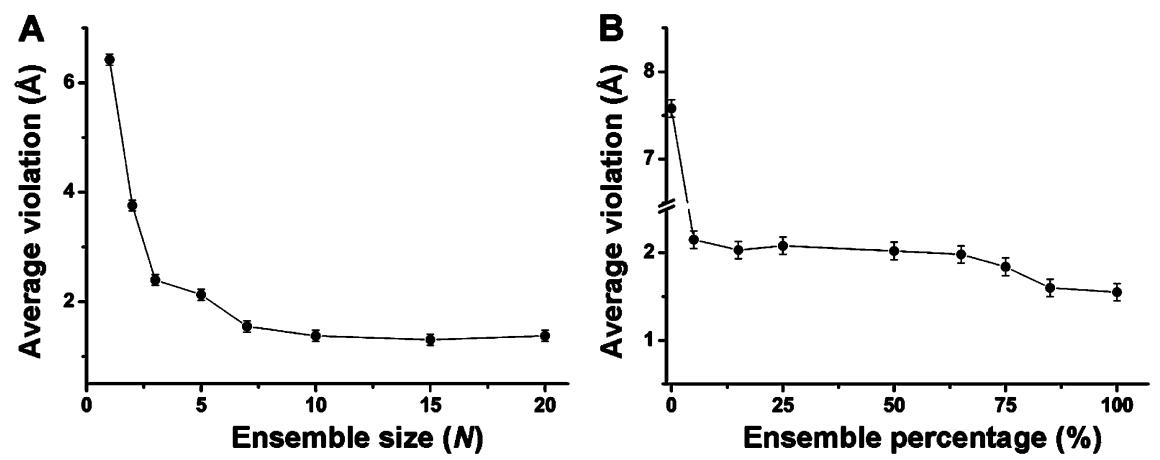

Figure 4. Plot of the average violation of all experimental distances versus the number $(N)$ of Pc copies used in ensemble docking (A) and versus the ensemble percentage included in the restraints for the calculations (B). Error bars represent $2 \times \mathrm{SD}$ of the average violations obtained from three independent calculations performed with $N=1+7$ and an encounter percentage of $50 \%\left(f_{1}=0.5\right)$.
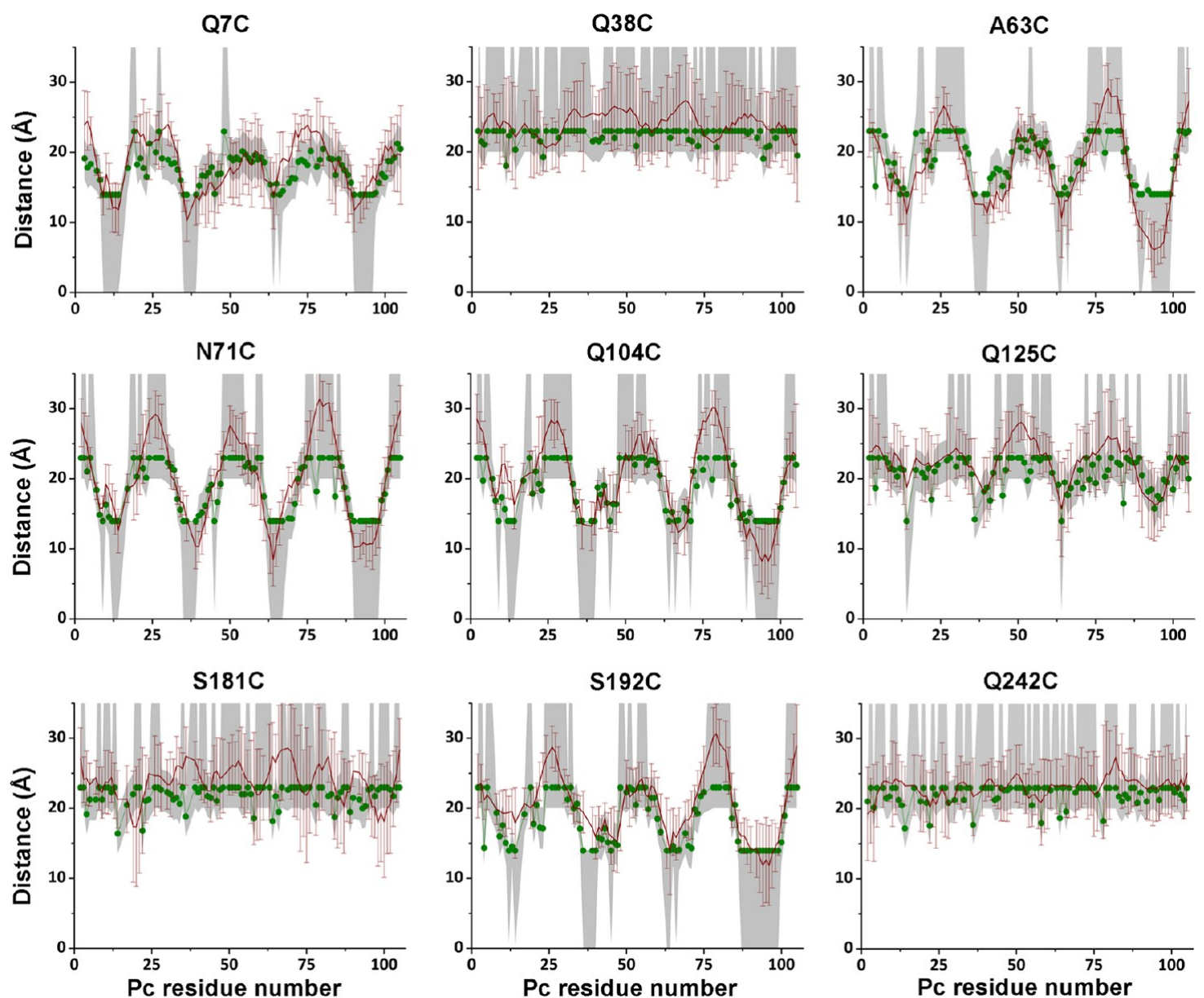

Figure 5. Ensemble docking. Experimental and back-calculated average distances between Pc amide protons and oxygen atoms of MTSL conjugated to Cyt $f$ are plotted against the Pc residue number. The green circles and lines represent the experimental distances, and the gray areas indicate the error margins. The average distances back-calculated from 20 ensembles are shown as a red line with error bars representing the SD. Calculations were performed with $N=7$ and $f_{1}=1.0$.

calculations. When the restraints derived either from variant Cyt $f$ A63-MTSL or from both variants Cyt $f$ Q38- and A63CMTSL were excluded from the calculations, the distribution and the fit of the experimental data did not improve, yielding average ensemble violation values of 1.9 and 1.8 , respectively, for $N=7$ and $f_{1}=1$ (see below). In our hands, converting PREs to distances worked best, probably because Pc approaches some spin labels closely. Due to inverse sixth power distance dependence, very small movements at short distance result in very large PRE changes that skew the outcome of the calculations. A repulsion function to avoid steric collision between Cyt $f$ and the Pc molecules was the only other interaction included in the calculations. Calculations were performed by varying the size $(N)$ of the docked ensemble, which ranged from 1 to 20 copies of Pc. The generated ensembles were evaluated by calculating the average violation over all experimental distances (see Experimental Section for details). As can be seen in Figure 4, a large decrease of the 
average violation was observed, going from a single copy of Pc up to $N=7$, while further increase of the number of $\mathrm{Pc}$ molecules $(N>7)$ did not improve the fitting. Thus, a combined docking of seven Pc copies simultaneously can produce a population distribution that can mostly satisfy the experimental PREs.

The solution structure of the final complex was previously reported, ${ }^{16}$ taking advantage of intermolecular PCS generated by the paramagnetic $\mathrm{Fe}^{\mathrm{III}}$ of Cyt $f$ on Pc nuclei. However, the results in Figure $4 \mathrm{~A}$ show that a single orientation cannot account for the PRE data. In a earlier study we showed that the model of the final complex is already insufficient to explain the PRE data of only three nearby spin labels. ${ }^{21}$ Thus, the PRE data describe a combination of the final state and encounter complex. Following this rationale, calculations were carried out with $N=1+7$, where 1 represents the final complex and 7 the number of copies in the ensemble. ${ }^{48}$ The contribution of the final complex to the experimental PREs was subtracted to obtain the PREs of the encounter state only, in analogy to what was done for the MC calculations. Thus, the back-calculated PREs from the solution model ${ }^{16}$ were subtracted from the experimental values, assuming a population of the final complex $\left(f_{2}\right)$ between 0 and 1 . The resulting PREs were converted to distances and used for ensemble docking. Figure 4B presents the average violation as a function of the fraction of encounter state $\left(f_{1}\right)$. The violations unequivocally indicate that the measured PREs do not derive from the final structure alone. Interestingly, a small fraction of encounter state is sufficient to decrease the average violation sharply. The average violation decreases slightly from 2.15 for $f_{1}=0.05$ encounter complex to 1.55 for $f_{1}=1$. In Figure 5 the results from the simulation carried out using seven conformers of $\operatorname{Pc}(N=7)$ and assuming a pure encounter state $\left(f_{1}=1\right)$ are shown.

The ensemble structures were used to back-calculate the distances between the oxygen atom of MTSL and Pc amide protons (red line), and these were compared with the experimental distances (green circles and line). The generated encounter complex fits the experimental data well, being within the error margins for most residues, although small deviations are observed for some residues at several spin label positions. The considerable standard deviations observed for the distances for the generated ensembles (error bars for red line) are noteworthy, because it is a clear illustration that rather different ensembles of seven Pc copies can fit the large experimental data set equally well, emphasizing the nature of the 'inverse problem' mentioned above.

Estimation of the Fraction of the Encounter Complex. The structure of the final complex was based on experimental PCS, not on PRE, and consists of a single orientation of Pc relative to Cyt $f$, so by reducing the contribution of the final complex, it is expected that it is easier to create an ensemble that matches the experimental PREs. Therefore, the small decrease of the average violation with increasing fraction of the encounter complex (Figure 4B) may not be significant, indicating that the PRE data cannot distinguish between a fraction of the encounter complex of $5 \%$ and $100 \%$. Since both PCS and PRE account for minor species present in solution, PCSs were back-calculated for the generated encounter complexes and compared with the experimental PCS data. To correlate experimental and back-calculated PCS, a $Q$ factor (eq S2) was calculated for different fractions of final structure $\left(f_{2}\right)$ (Figure S6). The size of the axial component of the magnetic susceptibility anisotropy $\left(\Delta \chi_{\mathrm{ax}}\right)$ of Cyt $f \mathrm{Fe}^{\mathrm{III}}$ is not known precisely, so a range of values was tested. For a final complex only $\left(f_{1}=0\right)$, the best fit of the PCS is found for $\Delta \chi_{\mathrm{ax}}$ values $<50 \%$ of the one derived from EPR data, in line with earlier findings. ${ }^{16}$ The low-lying excited states for a low-spin ferric heme explain why the $\mathrm{g}$ tensor at $10 \mathrm{~K}$ cannot readily be used to calculate the $\Delta \chi_{\mathrm{ax}}$ at $298 \mathrm{~K}$. For all but very low values of $\Delta \chi_{\mathrm{ax}}$, the combination of final complex and encounter ensemble (determined using the PRE ensemble docking) improves the fit between experimental and back-calculated PCS. Unfortunately, the lowest $Q$ value achievable is always about 0.1 (see Figure S6), and this minimum is found at increasing values of $f_{1}$ for larger $\Delta \chi_{\mathrm{ax}}$ values. Therefore, establishing the encounter state fraction on the basis of PCS is not possible as long as $\Delta \chi_{\mathrm{ax}}$ cannot be established. For comparison, $\Delta \chi_{\text {ax }}$ for Cyt $c$ is about $3.3 \times 10^{-32} \mathrm{~m}^{3} .52$ If $\Delta \chi_{\text {ax }}$ of Cyt $f$ would be the same, the fraction $f_{1}$ would be 0.25 .

Visualization of the Encounter Complex. To represent the encounter complex, an ensemble of 144 solutions, for a total of 1008 Pc molecules was generated $\left(N=7 ; f_{2}=0\right)$. As shown in Figure 6, Pc visits a large area of Cyt $f$. The density
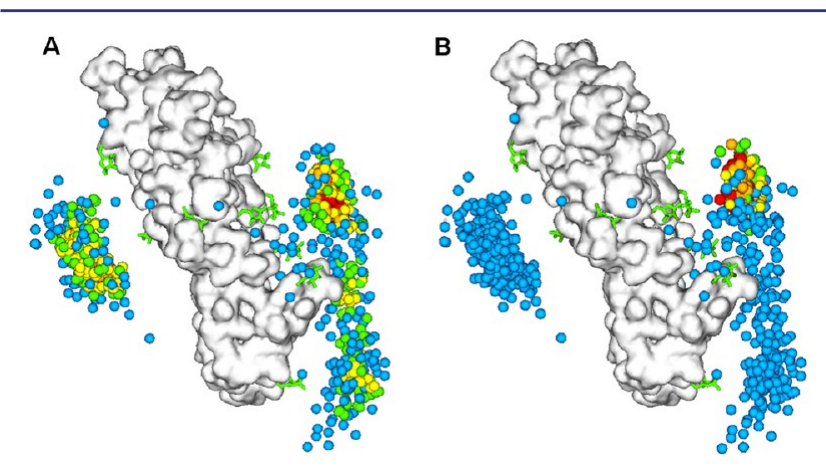

Figure 6. Encounter complex of the Pc-Cyt $f$ complex. Cyt $f$ is shown as a white surface and spin labels as green sticks. Pc centers-of-mass are represented by spheres. (A) Pc centers of mass are color-coded to indicate the density of the distributions, decreasing from red to blue. Densities were determined by counting the number of neighbors within $2.5 \AA$ A. (B) Pc centers-of-mass are color-coded to indicate the distance between $\mathrm{Cu}$ in $\mathrm{Pc}$ and $\mathrm{Fe}$ in Cyt $f$, increasing from red to blue (red $\leq 16 \AA$; orange $\leq 18 \AA$; yellow $\leq 20 \AA \AA$; green $\leq 22 \AA$; blue $>22$ A).

plot (Figure 6A) shows the centers-of-mass of Pc colored according to the density, with red and blue representing the most and least populated positions, respectively.

Two defined encounter locations can be distinguished. The first one is close to the binding site in the final complex, and the second on the other side of Cyt $f$, opposite to the binding site in the final complex. These locations are discussed further below. It should be noted that even with nine spin labels, it was not possible to sample the encounter state at all locations on the Cyt $f$ surface sufficiently. The cytochrome is a very elongated protein, and from the spin label positions in Figure 1 (central panel), the regions that were not sampled can be identified. It cannot be excluded that the area sampled in the encounter complex is still larger than the surface area covered in our experiments. Nevertheless, from the current analysis, it is obvious that $\mathrm{Pc}$ samples quite a significant fraction of the surface of its partner.

It is thought that in ET systems the formation of the encounter complex reduces the dimensionality of the search for the active site and increases the probability of ET. ${ }^{2}$ To determine which of the encounter complex orientations were 
compatible with rapid ET, the distances between $\mathrm{Cu}$, in $\mathrm{Pc}$, and $\mathrm{Fe}$, in Cyt $f$, were calculated, and the centers-of-mass of Pc were color-coded accordingly (Figure 6B). Structures with $\mathrm{Cu}-\mathrm{Fe}$ distance $\leq 16 \AA$ (red dots), thus in principle suitable for fast $\mathrm{ET}^{53}$ are exclusively located in front of the heme, in the vicinity of the final structure.

\section{DISCUSSION}

The structure of $\mathrm{Pc}-\mathrm{Cyt} f$ final complex from the cyanobacterium Nostoc sp. PCC7119 was solved by NMR on the basis of PCS data. ${ }^{16}$ The results were consistent with a conformation that accounts for the ET reaction between the two proteins. A recent PRE study ${ }^{21}$ on the same system demonstrated that the complex exists partly in a dynamic ensemble of orientations. That study was limited by the number and location of the spin labels, which were close to the site of the specific binding of Pc. It inspired the current study, in which we characterized the encounter complex of Pc and Cyt $f$ by attaching the spin label MTSL to nine sites on Cyt $f$. MTSL is a small hydrophobic molecule, and its presence in particular regions of the protein could influence the complex formation with the interaction partner. Some interference by the spin label in the Pc-Cyt $f$ complex formation was observed only at the position Q38 and A63. The exclusion of the data either from one (A63) or both (Q38 and A63) the spin labels had little influence on the results. PREs were mainly observed for spin labels situated near to the binding site indicated by the PCSbased model, although effects were also measured for the remaining spin label positions. The detection of widespread PREs clearly indicates that Pc samples a large surface area. The similarity of PRE patterns observed in the presence of spin labels close to the binding site (Figure 3 ) suggests that Pc is approaching Cyt $f$ with the residues forming the hydrophobic patch and the region around R93. Independent of the location of the paramagnetic probes, the residues that experience the strongest PREs reside in these regions, implying that $\mathrm{Pc}$ is always oriented in the same way toward Cyt $f$.

The general model of protein association states that the formation of the encounter complex is an electrostatically driven process. ${ }^{2}$ On the basis of this assumption, computational approaches, such as BD and MC docking, have been developed to describe the encounter complex as end-point of electrostatic steering toward the interacting partners. ${ }^{26,54}$ These methods successfully described the encounter complex in cases of protein association guided by charge-charge interactions. $^{6-8,54-56}$ The MC simulations of the Pc-Cyt $f$ encounter complex did not produce a result in accordance with the experimental data. It is important to note that despite the overall electrostatic potential of $\mathrm{Pc}$ and Cyt $f$ being positive and negative, respectively, Pc shows a defined charge distribution, but Cyt $f$ has a diffuse surface charge. For interaction partners with weak charge complementarity, it has been demonstrated that electrostatics do not play an exclusive role in protein association, but desolvation is the main driving force in binding. ${ }^{9}$ Furthermore, the interface of the encounter complex can bury a significant solvent-accessible surface area, suggesting a role for hydrophobic interactions in the stabilization of the encounter complex. ${ }^{57}$ In the Pc-Cyt $f$ complex from the cyanobacterium $\mathrm{Ph}$. laminosum, the association is dominated by hydrophobic interactions, and only hydrophobic contacts stabilize the final complex. ${ }^{12}$ In the Nostoc Pc-Cyt $f$ system, the specific binding interface and the putative ET sites on both proteins comprise hydrophobic regions, ${ }^{16}$ similar in size and composition to the Ph. laminosum counterparts, but electrostatic forces play a significant role in the association reaction $^{18,19}$ and in the orientation of the final complex. ${ }^{43}$ Since already in the encounter complex Pc is oriented toward Cyt $f$ with its hydrophobic patch, we propose that during the initial stage of the encounter complex formation, long-range electrostatics preorient Pc toward Cyt $f$, and hydrophobic interactions keep Pc close to the surface of Cyt $f$ and help to stabilize the encounter state.

The simulation of the encounter complex on the basis of the experimental PREs resulted in two distinct encounters: One is located at the side of the specific binding surface of Cyt $f$ and the other one at the opposite side. In Figure 7 the encounter

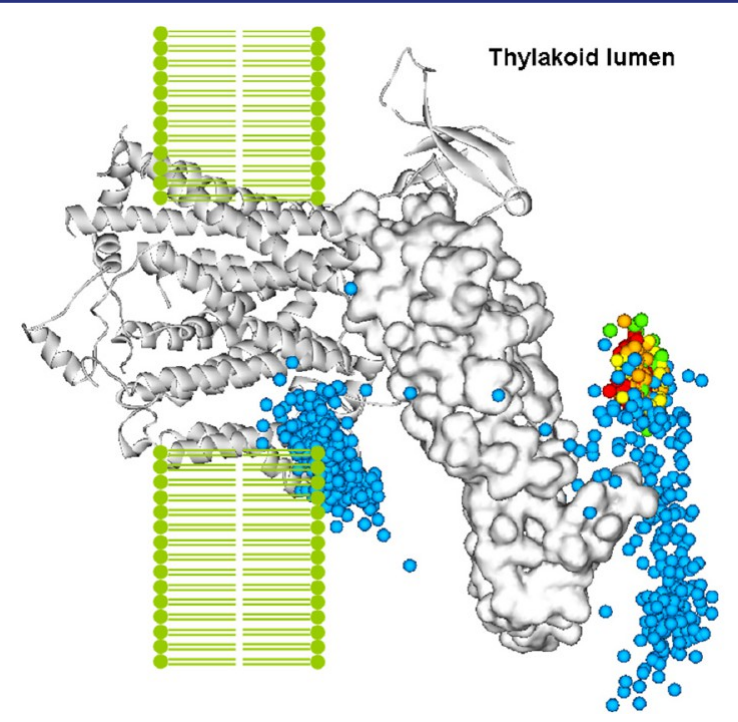

Figure 7. Encounter complex of the Pc-Cyt $f$ complex superimposed on the structure of the cytochrome $b_{6} f$ complex (PDB entry 2ZT9), embedded in the thylakoid membrane. Cytochrome $b_{6} f$ complex is a dimer, but only a monomer is shown. Cyt $f$ is shown as a white surface, and $\mathrm{Pc}$ centers-of-mass are represented by spheres, which are colorcoded to indicate the distance between $\mathrm{Cu}$ in $\mathrm{Pc}$ and $\mathrm{Fe}$ in $\mathrm{Cyt} f$, as in Figure 6B. The remaining components of the $b_{6} f$ complex are shown as ribbons.

complex is superimposed with the cytochrome $b_{6} f$ complex, as it is found in the thylakoid membrane, with the Pc centers-ofmass colored on the bases of the $\mathrm{Cu}-\mathrm{Fe}$ distance.

The encounter complex located opposite to the ET site places Pc inside the thylakoid membrane. The Cyt $f$ soluble part was shown to be only lightly flexible in the thylakoid lumen, ${ }^{58}$ suggesting that Pc cannot interact with Cyt $f$ at this site under the physiological conditions. The observation of encounters at this site is likely an artifact due to the use of the soluble part of Cyt $f$ in isolation and not embedded in the thylakoid membrane. The encounters located in front of the binding site diffusely extends from the heme to the end of the small domain of Cyt $f$. In other studies, diffusive encounter complexes have also been described. ${ }^{59}$ Either the partners sample large areas or form encounters at several more defined regions on the surface of the partner. ${ }^{3,4,60,61}$ Due to the diffuse distribution of negative charges on Cyt $f$, Pc probes a large part of the surface, instead of being attracted to specific sites by localized charges.

The formation of an encounter complex in many cases does not lead to a productive association, hence fruitful and futile encounter complexes can be distinguished. ${ }^{3}$ The encounter 


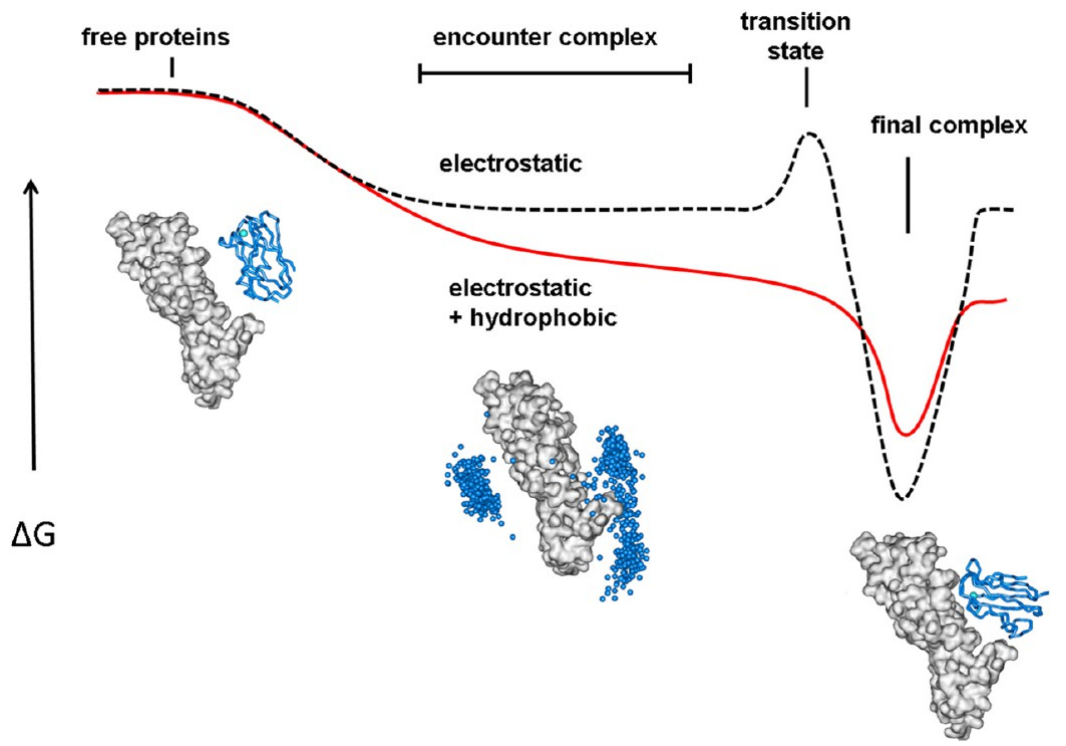

Figure 8. Free energy diagrams of proteins association pathways with high (dashed black line) and no (solid red line) energy barrier for the transition state from the encounter to the final complex.

ensemble shows a high density in the region directly in front of the heme, suitable for ET (red dots in Figures 6B and 7). These orientations can obviously be considered fruitful encounters. For many other encounters, the distances between the redox centers are unfavorable for ET, but not all of these are futile. The further away from the ET active site Pc binds, the less chance it has to diffuse to an ET active orientation before dissociation from Cyt $f$. Thus, the distinction between fruitful and futile encounter is a gradual one, and it is difficult to tell when these complexes can rearrange into a productive orientation.

The population of the encounter state, in relation with the final state population, significantly varies among different complexes. In some non-ET complexes the population of the encounter state has been determined to be $10 \%,{ }^{23,57}$ and in the ET complex Cyt $c-$ Cyt $c$ peroxidase, it has been shown to be $30 \%{ }^{8}$ For Pc-Cyt $f$ complex from Nostoc, it was not possible to establish the fraction accurately. Complexes that only exist in the encounter state have also been described for myoglobin-Cyt $b_{5}{ }^{44}$ and adrenodoxin-Cyt $c .{ }^{60}$ In Nostoc, the existence of the complex as merely an encounter state seems unrealistic. The size of CSP in the wild-type complex ${ }^{16}$ and in the presence of spin labeled Cyt $f$ also in this study is indicative of the formation of a stereospecific complex. Moreover, PCS from the heme generated a converged structure stabilized in a defined orientation, ${ }^{16}$ in which hydrophobic contacts and electrostatic interactions are optimized within the structure. The sensitivity of this approach to lowly populated states is limited, but it clearly demonstrates the existence of a final state.

At the same time, the diffuse nature of the encounter complex on Cyt $f$ surface suggests that in this system a final orientation may not be a fundamental requirement for the functionality of the complex. In fact, the efficient turnover required for rapid ET through the photosynthetic redox chain ${ }^{62}$ precludes the formation of a tight complex and favors the conditions for the existence of the ET active complex in multiple orientations ${ }^{63}$ to enhance the probability of $\mathrm{ET}^{2}$ Against this background, the finding that hydrophobic contacts play a role not only in the final complex but also in the encounter complex is interesting. It blurs the distinction between both states and would allow for a smooth transition from encounter to final complex via a gradual optimization of the hydrophobic contacts in the interface (Figure 8, solid line). This model of protein complex formation allows for more rapid formation of the final complex than in the case of a model with an activation energy barrier between both states (Figure 8, dashed line), used for other protein complexes. ${ }^{3}$ The interface area of Cyt $f$ in encounter complex at the 'front side' of Cyt $f$ comprises two hydrophobic patches: one located in the big domain (Y1, F3, W4, A63, V68, P118, L119, P120, Y161, and P162), and the other in the small domain (A184, L196, V197, V206, V207, P212, and A213). To test the proposed model, it could be of interest to mutate these residues to polar ones or smaller hydrophobic site chains. Substitution of residues that are only part of the encounter complex is expected to reduce the association rate, whereas mutations close to the heme can disrupt the formation of ET active complexes, reducing the ET rate and shifting the balance toward the non-ET active conformations.

\section{CONCLUSIONS}

The combination of paramagnetic NMR spectroscopy and theoretical calculations has enabled the characterization of the encounter complex of Cyt $f$ and Pc. With nine spin label positions it was shown that the encounter complex is extended over a large area of Cyt $f$ and even includes futile complexes far from the binding site, which are probably nonphysiological. It serves as a reminder that the natural context must be considered when studying the extent of surface areas sampled in the encounter complex. The MC docking calculations indicate that charge interactions play a role but are not dominant; instead hydrophobic contacts appear to guide Pc to the ET active positions on Cyt $f$. We speculate that the hydrophobic interactions in the encounter complex may ensure a relative flat energy landscape during all phases of association, without a clear distinction between the encounter and the active complexes. A flat energy landscape ensures rapid transitions between all states, which is relevant for systems that do not require a high degree of specificity, such as ET complexes. 


\section{ASSOCIATED CONTENT}

\section{S Supporting Information}

Description of the primers used for Cyt $f$ site-directed mutagenesis; binding curves and CSP maps of $\mathrm{Zn}$-substituted Pc in the presence of Cyt $f$; representation of the MC-simulated Pc-Cyt $f$ encounter complex; plots of the experimental and back-calculated distances from the MC-simulated Pc-Cyt $f$ encounter; $Q$ factors for experimental and back-calculated PCS. This material is available free of charge via the Internet at http://pubs.acs.org.

\section{AUTHOR INFORMATION}

\section{Corresponding Author}

m.ubbink@chem.leidenuniv.nl

\section{Notes}

The authors declare no competing financial interest.

\section{ACKNOWLEDGMENTS}

S.S. kindly acknowledges Maryam Hashemi Shabestari and Martin van Son for EPR measurements. S.S. and M.U. received financial support from The Netherlands Organisation for Scientific Research (NWO), grant 700.57.011 (S.S.) and 700.58.441 (M.U.). J.F. was supported by DAAD for the Erasmus Exchange Programme. G.M.U. and J.M.F. were supported by the German Science Foundation (DFG; GRK 1640).

\section{REFERENCES}

(1) Schreiber, G. Curr. Opin. Struct. Biol. 2002, 12, 41-47.

(2) Ubbink, M. FEBS Lett. 2009, 583, 1060-1066.

(3) Harel, M.; Spaar, A.; Schreiber, G. Biophys. J. 2009, 96, 42374248

(4) Fawzi, N. L.; Doucleff, M.; Suh, J. Y.; Clore, G. M. Proc. Natl. Acad. Sci. U.S.A. 2010, 107, 1379-1384.

(5) Schreiber, G.; Fersht, A. R. Nat. Struct. Biol. 1996, 3, 427-431.

(6) Gabdoulline, R. R.; Wade, R. C. Biophys. J. 1997, 72, 1917-1929.

(7) Northrup, S. H.; Boles, J. O.; Reynolds, J. C. Science 1988, 241, 67-70.

(8) Bashir, Q.; Volkov, A. N.; Ullmann, G. M.; Ubbink, M. J. Am. Chem. Soc. 2010, 132, 241-247.

(9) Camacho, C. J.; Weng, Z.; Vajda, S.; DeLisi, C. Biophys. J. 1999, 76, 1166-1178.

(10) Camacho, C. J.; Vajda, S. Proc. Natl. Acad. Sci. U.S.A. 2001, 98, 10636-10641.

(11) Sugase, K.; Dyson, H. J.; Wright, P. E. Nature 2007, 447, 10211025

(12) Crowley, P. B.; Otting, G.; Schlarb-Ridley, B. G.; Canters, G. W.; Ubbink, M. J. Am. Chem. Soc. 2001, 123, 10444-10453.

(13) Gorman, D. S.; Levine, R. P. Proc. Natl. Acad. Sci. U.S.A. 1965, 54, 1665-1669.

(14) Ubbink, M.; Ejdeback, M.; Karlsson, B. G.; Bendall, D. S. Structure 1998, 6, 323-335.

(15) Lange, C.; Cornvik, T.; Diaz-Moreno, I.; Ubbink, M. Biochim. Biophys. Acta 2005, 1707, 179-188.

(16) Diaz-Moreno, I.; Diaz-Quintana, A.; De la Rosa, M. A.; Ubbink, M. J. Biol. Chem. 2005, 280, 35784.

(17) Hulsker, R.; Baranova, M. V.; Bullerjahn, G. S.; Ubbink, M. J. Am. Chem. Soc. 2008, 130, 1985-1991.

(18) Albarran, C.; Navarro, J. A.; Molina-Heredia, F. P.; Murdoch, P. S.; De la Rosa, M. A.; Hervas, M. Biochemistry 2005, 44, 11601-11607.

(19) Albarran, C.; Navarro, J.; De la Rosa, M. A.; Hervas, M. Biochemistry 2007, 46, 997-1003.

(20) Gross, E. L.; Rosenberg, I. Biophys. J. 2006, 90, 366-380.

(21) Scanu, S.; Förster, J.; Finiguerra, M. G.; Shabestari, M. H.; Huber, M.; Ubbink, M. ChemBioChem 2012, 13, 1312-1318.
(22) Iwahara, J.; Clore, G. M. Nature 2006, 440, 1227-1230.

(23) Tang, C.; Iwahara, J.; Clore, G. M. Nature 2006, 444, 383-386.

(24) Suh, J. Y.; Tang, C.; Clore, G. M. J. Am. Chem. Soc. 2007, 129, 12954-12955.

(25) Clore, G. M.; Iwahara, J. Chem. Rev. 2009, 109, 4108-4139.

(26) Ullmann, G. M.; Knapp, E. W.; Kostic, N. M. J. Am. Chem. Soc. 1997, 119, 42-52.

(27) Milikisyants, S.; Scarpelli, F.; Finiguerra, M. G.; Ubbink, M.; Huber, M. J. Magn. Reson. 2009, 201, 48-56.

(28) Arslan, E.; Schulz, H.; Zufferey, R.; Kunzler, P.; Thony-Meyer, L. Biochem. Biophys. Res. Commun. 1998, 251, 744-747.

(29) Delaglio, F.; Grzesiek, S.; Vuister, G. W.; Zhu, G.; Pfeifer, J.; Bax, A. J. Biomol. NMR 1995, 6, 277-293.

(30) Vranken, W. F.; Boucher, W.; Stevens, T. J.; Fogh, R. H.; Pajon, A.; Llinas, M.; Ulrich, E. L.; Markley, J. L.; Ionides, J.; Laue, E. D. Proteins 2005, 59, 687-696.

(31) Battiste, J. L.; Wagner, G. Biochemistry 2000, 39, 5355-5365.

(32) Baniulis, D.; Yamashita, E.; Whitelegge, J. P.; Zatsman, A. I.; Hendrich, M. P.; Hasan, S. S.; Ryan, C. M.; Cramer, W. A. J. Biol. Chem. 2009, 284, 9861-9869.

(33) Schmidt, L.; Christensen, H. E.; Harris, P. Acta Crystallogr., Sect. D:Biol. Crystallogr 2006, 62, 1022-1029.

(34) Brunger, A. T.; Karplus, M. Proteins 1988, 4, 148-156.

(35) Brooks, B. R.; Bruccoleri, R. E.; Olafson, B. D.; States, D. J.; Swaminathan, S.; Karplus, M. J. Comput. Chem. 1983, 4, 187-217.

(36) MacKerell, A. D.; Bashford, D.; Bellott; Dunbrack, R. L.; Evanseck, J. D.; Field, M. J.; Fischer, S.; Gao, J.; Guo, H.; Ha, S.; Joseph-McCarthy, D.; Kuchnir, L.; Kuczera, K.; Lau, F. T. K.; Mattos, C.; Michnick, S.; Ngo, T.; Nguyen, D. T.; Prodhom, B.; Reiher, W. E.; Roux, B.; Schlenkrich, M.; Smith, J. C.; Stote, R.; Straub, J.; Watanabe, M.; Wiorkiewicz-Kuczera, J.; Yin, D.; Karplus, M. J. Phys. Chem. B 1998, 102, 3586-3616.

(37) Baker, N. A.; Sept, D.; Joseph, S.; Holst, M. J.; McCammon, J. A. Proc. Natl. Acad. Sci. U.S.A. 2001, 98, 10037-10041.

(38) Metropolis, N.; Ulam, S. J. Am. Stat. Assoc. 1949, 44, 335-341.

(39) Iwahara, J.; Schwieters, C. D.; Clore, G. M. J. Am. Chem. Soc. 2004, 126, 12800-12808.

(40) García de la Torre, J.; Huertas, M. L.; Carrasco, B. J. Magn. Reson. 2000, 147, 138-146.

(41) Schwieters, C. D.; Kuszewski, J. J.; Tjandra, N.; Clore, G. M. J. Magn. Reson. 2003, 160, 65-73.

(42) Ubbink, M.; Lian, L. Y.; Modi, S.; Evans, P. A.; Bendall, D. S. Eur. J. Biochem. 1996, 242, 132-147.

(43) Diaz-Moreno, I.; Diaz-Quintana, A.; De la Rosa, M. A.; Crowley, P. B.; Ubbink, M. Biochemistry 2005, 44, 3176-3183.

(44) Worrall, J. A. R; Liu, Y. J.; Crowley, P. B.; Nocek, J. M.; Hoffman, B. M.; Ubbink, M. Biochemistry 2002, 41, 11721-11730.

(45) Volkov, A. N.; Bashir, Q.; Worrall, J. A. R.; Ullmann, G. M.; Ubbink, M. J. Am. Chem. Soc. 2010, 132, 11487-11495.

(46) Tang, C.; Schwieters, C. D.; Clore, G. M. Nature 2007, 449, $1078-1082$.

(47) Tang, C.; Ghirlando, R.; Clore, G. M. J. Am. Chem. Soc. 2008, $130,4048-4056$.

(48) Tang, C.; Louis, J. M.; Aniana, A.; Suh, J. Y.; Clore, G. M. Nature 2008, 455, 693-696.

(49) Fawzi, N. L.; Doucleff, M.; Suh, J. Y.; Clore, G. M. Proc. Natl. Acad. Sci. U.S.A. 2010, 107, 1379-1384.

(50) Anthis, N. J.; Doucleff, M.; Clore, G. M. J. Am. Chem. Soc. 2011, 133, 18966-18974.

(51) Volkov, A. N.; Ubbink, M.; van Nuland, N. A. J. J. Biomol. NMR 2010, 48, 225-236.

(52) Worrall, J. A. R.; Kolczak, U.; Canters, G. W.; Ubbink, M. Biochemistry 2001, 40, 7069-7076.

(53) Moser, C. C.; Keske, J. M.; Warncke, K.; Farid, R. S.; Dutton, P. L. Nature 1992, 355, 796-802.

(54) Gabdoulline, R. R.; Wade, R. C. J. Mol. Recognit. 1999, 12, 226234.

(55) Gabdoulline, R. R.; Wade, R. C. J. Mol. Biol. 2001, 306, 11391155. 
(56) Spaar, A.; Dammer, C.; Gabdoulline, R. R.; Wade, R. C.; Helms, V. Biophys. J. 2006, 90, 1913-1924.

(57) Kim, Y. C.; Tang, C.; Clore, G. M.; Hummer, G. Proc. Natl. Acad. Sci. U.S.A. 2008, 105, 12855-12860.

(58) Hasan, S. S.; Cramer, W. A. Phys. Chem. Chem. Phys. 2012, 14, 13853-13860.

(59) Harel, M.; Cohen, M.; Schreiber, G. J. Mol. Biol. 2007, 371, $180-196$.

(60) Xu, X. F.; Reinle, W. G.; Hannemann, F.; Konarev, P. V.; Svergun, D. I.; Bernhardt, R.; Ubbink, M. J. Am. Chem. Soc. 2008, 130, 6395-6403.

(61) Liang, Z. X.; Nocek, J. M.; Huang, K.; Hayes, R. T.; Kurnikov, I. V.; Beratan, D. N.; Hoffman, B. M. J. Am. Chem. Soc. 2002, 124, 68496859.

(62) Hope, A. B. Biochim. Biophys. Acta 2000, 1456, 5-26.

(63) Crowley, P. B.; Ubbink, M. Acc. Chem. Res. 2003, 36, 723-730. 